\title{
Research on Electric Energy Meter Technology in the Background of Smart Grid Based on Particle Swarm Optimization Algorithm
}

\author{
Ning Li ${ }^{1}$, Yin Chang Zhang ${ }^{1}$,Liang Zhang ${ }^{1}$,Jun Sun ${ }^{2}$ \\ 1 State Grid Xinjiang Electric Power Co., Ltd. Electric Power Research Institute Urumqi, Xinjiang \\ Uygur Autonomous Region 830000 \\ 2 Wuhan Pandian Electrical Technology Co., Ltd \\ Wuhan, Hubei province 430108
}

Keywords: Binary ant colony optimization algorithm; Smart grid; ADE7758; Smart meter

\begin{abstract}
With the continuous development of China's power electronics technology, the power sector's optimization and control efforts for various energy consuming manufacturing enterprises have also been significantly increased. At the same time, the energy problem has been seen as an urgent need to optimize the use of. The power parameters measured by traditional electric power monitoring instruments are too single. Compared with the increasingly complex power supply network, the overall monitoring of the power quality cannot be achieved. In view of this, in the background of smart grid based on binary ant colony optimization algorithm, a kind of power monitoring instrument based on ADE7758 is proposed in this paper. Compared with the previous power monitoring instrument, the central processor adopts ARM9 chip, which has fast processing speed, high precision of data, supports a variety of communication interfaces and the peripheral expansion function is strong.
\end{abstract}

\section{Introduction}

Demand-side management is a basic and important function of modern power grid dispatching and control. It fundamentally changes the traditional thinking mode of simply focusing on increasing energy supply to meet the growth of energy demand. Instead, it uses the energy saved on the demand side as a supply. The concept of alternative resources can be used to improve the efficiency of the use of systems and resources and has an important strategic role in the power industry and economic development and environmental protection. Its implementation can excavate terminal energy-saving capability to a great extent, and it is a necessary means and a powerful guarantee for achieving energy-saving and emission-reduction and improving efficiency ${ }^{[1]}$. At the same time, the outstanding feature of the smart grid is to use advanced information technologies to flexibly integrate and dispatch demand-side resources, achieve two-way interaction between information and electricity, and predict that there will be considerable progress and development in the future of the smart grid era. The smart meter is an intelligent terminal of the smart grid, which is not a traditional electric energy meter. In addition to the measurement function of the basic electricity consumption of the traditional electric energy meter, the smart meter also has two-way multi-rate metering functions, user-end control functions, bidirectional data communication functions for multiple data transmission modes, and anti-tampering functions to adapt to the use of smart grids and new energy. Smart meters represent future energy-saving smart grid end-user intelligent terminal development direction.

\section{Application of Binary Particle Swarm Optimization Algorithm in Demand Side Scheduling of Smart Grid}

Load management refers to the development of a deterministic or time-varying policy to stimulate the load to respond in time and adjust the load when the system reliability is affected or the electricity price is high. Interruptible load is a kind of load management method that interrupts partial load power supply after the user responds by sending an interrupt request signal to the user 
in the peak period of power demand according to the prior contract agreement between the supplier and the buyer.

This paper uses BPSO to perform interruptible load scheduling. Each particle Sch is a $19 \times 16$ matrix. Rows and columns correspond to the numbers and periods of interruptible loads, respectively ${ }^{[2]}$. If $\mathrm{n}$ is interrupted in time $\mathrm{t}$, then $\operatorname{Sch}(n, t)=1$, otherwise $\operatorname{Sch}(n, t)=0$. The speed of each iteration is:

$$
\begin{aligned}
V(n, t)^{(k+1)}= & V(n, t)^{(k)}+\phi \cdot \operatorname{rand}() \cdot\left(\operatorname{Sch}_{p}(n, t)-\operatorname{Sch}(n, t)^{(k)}\right) \\
& +\phi \cdot \operatorname{rand}() \cdot\left(\operatorname{Sch}_{g}(n, t)-\operatorname{Sch}(n, t)^{(k)}\right)
\end{aligned}
$$

The velocity is transformed into probability by using Sigmoid function, and then discretized by comparison with the random number. The initial state of the simulation is that all particles are distributed randomly in the solution space and satisfy the two-item distribution. The probability distribution of 0 and 1 values is $50 \%$ and $50 \%$.

The scheduling problem is actually a constrained multi-objective optimization problem. It considers the various security constraints and achieves the best economic results. The goal of this paper is to minimize disruptive scheduling and minimize the number of executions. And the constraints of the interruption capacity and load characteristics required per hour should be satisfied, that is, the actual interruption time of each load is less than the longest blackout time, and the actual continuous online time of each load is greater than the shortest sustained power

$$
\begin{aligned}
\operatorname{Fitness}(P) & =\sum_{t=1}^{T} \sum_{n=1}^{N} \operatorname{Sch}(n, t) \cdot P_{n} \cdot \lambda_{n} \\
& +\left(C_{\mathrm{int}} \cdot 2^{0} \cdot F I_{2}+C_{\mathrm{int}} \cdot 2^{1} \cdot F I_{3}+\ldots .+C_{\mathrm{int}} \cdot 2^{\beta-2} \cdot F I_{\beta}\right)
\end{aligned}
$$

Constraints:

$$
\begin{gathered}
\sum_{n=1}^{N} \operatorname{Sch}(n, j) \cdot P_{n} \geq \operatorname{Re} q(j) \\
N_{-} O F F(i) \leq M a x \_O F F(i) \\
N_{-} \text {ON }(i) \leq M i n \_O N(i)
\end{gathered}
$$

In the form:

$\operatorname{Sch}(\mathrm{n}, \mathrm{t})$ is the state of the $\mathrm{n}$ interruptible load in the $\mathrm{t}$ time;

$P_{n}$ is the capacity of the $\mathrm{n}$ load;

$\lambda_{n}$ is the cost of the interruption capacity of the $\mathrm{n}$ load unit;

$C_{\text {int }}$ is the weight of again interruption compensation, set to 1000 ;

$F I_{i}$ is the number of loads with interruption times greater than i;

$\beta$ is the maximum number of interruptions;

$\operatorname{Re} q(j)$ is the interruption capacity required for the $\mathrm{j}$ time;

$M a x \_O F F(i)$ is the longest power outage time of the i load;

Min_ON(i) is the shortest continuous power supply time for the i load;

$N_{-} \bar{O} F F(i)$ is the actual interruption time of the $\mathrm{i}$ load;

$N_{-} O N(i)$ is the actual continuous online time of the I load;

Combining two targets and two constraints, the constrained optimization problem is converted into an unconstrained optimization problem, and a single objective function is formed, which will constitute a single objective function for the cost of load, the cost of interruption, the penalty of insufficient interruption and the sum of the violation of the constraint fines.

$$
\begin{aligned}
\text { Fitness }(P) & =\sum_{t=1}^{T} \sum_{n=1}^{N} \operatorname{Sch}(n, t) \cdot P_{n} \cdot \lambda_{n} \\
& +\left(C_{\mathrm{int}} \cdot 2^{0} \cdot F I_{2}+C_{\mathrm{int}} \cdot 2^{1} \cdot F I_{3}+\ldots+C_{\mathrm{int}} \cdot 2^{\beta-2} \cdot F I_{\beta}\right) \\
& +P_{e v} \cdot V \\
& +P_{e u c} \cdot U C
\end{aligned}
$$

In the form:

$P_{e v}$ is the penalty weight of the number of units violating the load constraint;

$P_{e u c}$ is the penalty weight of insufficient interruption capacity per unit time; 
$V$ is the number of times that violate the load constraint;

$U C$ is the number of hours that are short of interruption.

The total cost is equal to the sum of the product of each load interruption capacity and the corresponding interruption cost, and the number of interruptions can be reduced by providing penalty measures. When the interrupted load is interrupted again, the economic penalty is generated, and the penalty $C_{\text {int }}$ doubles as the number of interruptions increases, and the number of interrupts can be minimized by such a constraint.

\section{Design of Smart Meter Based on ADE7758}

\section{Power Parameter Model}

The first is the calculation of active power, which is defined as the rate of electric energy flowing from the power to the load. It is determined by the voltage and current waveforms. The resulting waveform is called the instantaneous power signal, which is equal to the instantaneous power flow rate at any instant. The unit of power is Watt, or coke / second. The formula (7) shows the expression of the instantaneous power signal in the AC system ${ }^{[3]}$.

$$
\begin{gathered}
i(t)=\sqrt{2} \times V R M S \times \sin (w t) \\
i(t)=\sqrt{2} \times I R M \& \sin (
\end{gathered}
$$

VRMS is an effective value voltage, and IRMS is an effective value current.

$$
\begin{aligned}
& p(t)=v(t) \times i(t) \\
& p(t)=I R M S \times V R M S-I R N M S \times N V R M S \times \cos (2 w t)
\end{aligned}
$$

The integral average power calculation formula of the line cycle (n) is as follows:

$$
p=\frac{1}{n T} \int_{0}^{n T} p(t) d t=V R M S \times I R M S
$$

$\mathrm{t}$ is the signal period, and $\mathrm{p}$ refers to active or real power. In formula 9 , we can notice that the active power is equal to the DC component of the instantaneous power signal $p(t)$, that is, $V R M S \times I R M S$. This is the relationship between the active power calculated by the measured phases.

\section{Hardware Design}

Figure 1 shows the overall design of the system. The following analysis will focus on the main module design.

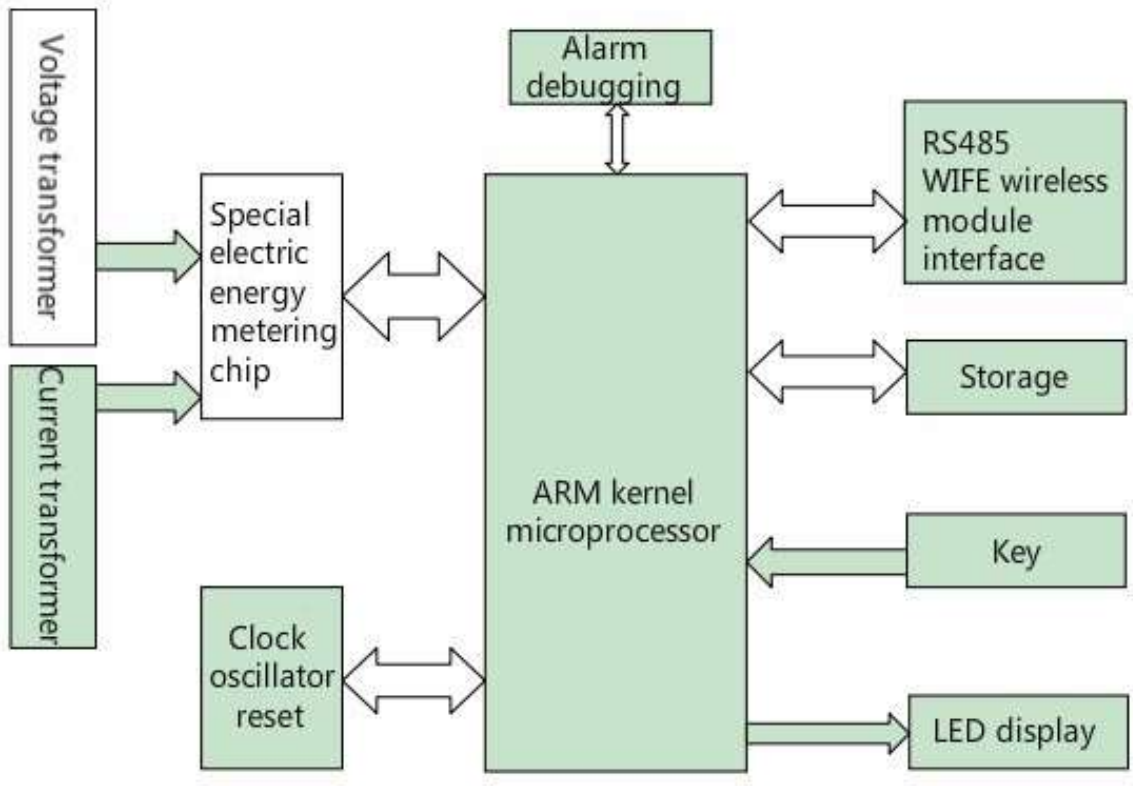

Figure 1. Overall system structure 
(1) Electric energy metering module

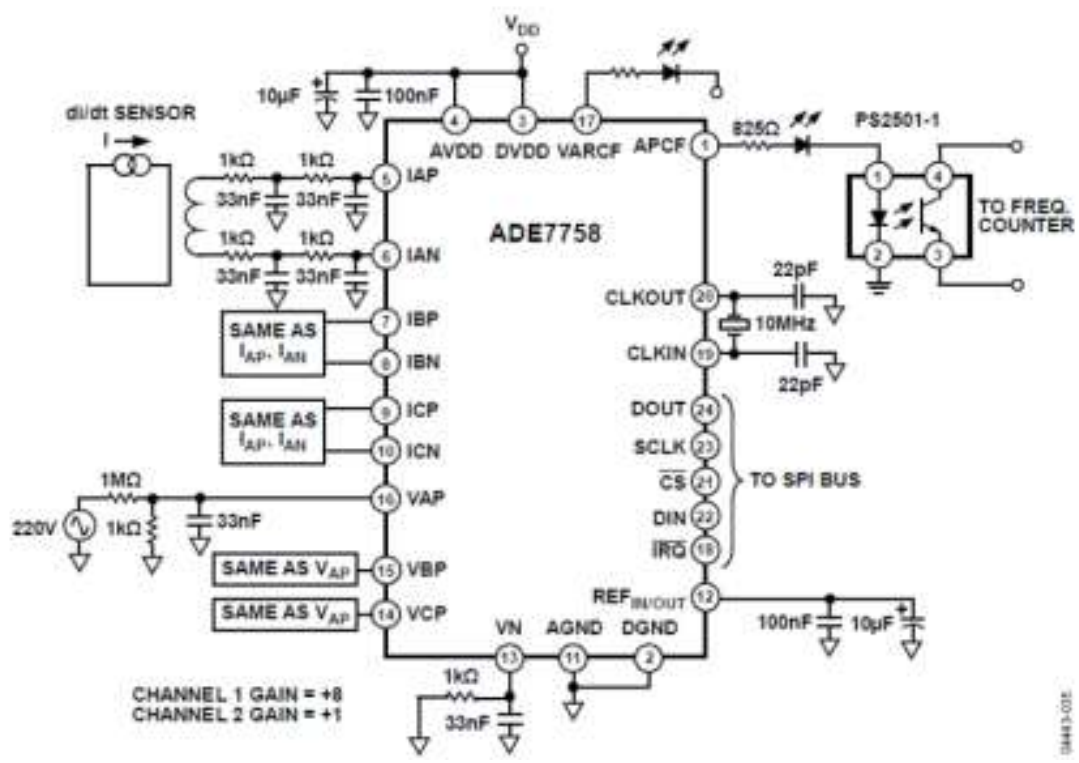

Figure.2 Typical application connection diagram of ADE7758 power chip

ADE7758 is a high accuracy three-phase power metering chip with embedded data processing capability. It has two pulse output functions and a serial interface. ADE7758 has six analog signal input ports, divided into current input and voltage input, as shown in Figure 2. The current input has 3 pairs of differential voltages, IAP and IAN, IBP and IBN, ICP and ICN. The maximum signal voltage difference of 3 current ports is $\pm 0.5 \mathrm{~V}$, and the current input terminal has a programmable gain amplifier (PGA), and the gain of the amplifier can be selected as 1,2 and 4. The voltage input port has three single ports: VAP, VBP and VCP. Compared with VN, these voltage input terminals have a range of $\pm 0.5 \mathrm{~V}$. Both the current and voltage channels have a programmable gain amplifier. After the analog electrical signal is connected to the corresponding pin of the ADE7758 chip, the gain amplification process, the analog digital conversion process, the voltage and current value are calibrated, and the values obtained after the filtering are stored in the register of the corresponding current and voltage effective values. The active power of the measured circuit is calculated.

(2) Microprocessor

The microprocessor is the core of the whole smart meter control and management. S3C2410 is mainly for handheld devices and applications with high cost performance and low power consumption. It is internally integrated with 3 channel UART ports, an IIC bus interface and 2 SPI interfaces. In addition, there are 4 timers with PWM functions, an internal clock, a watchdog timer and a RTC real-time clock. The basic peripheral circuit configuration of the system microprocessor is shown in Figure 3.

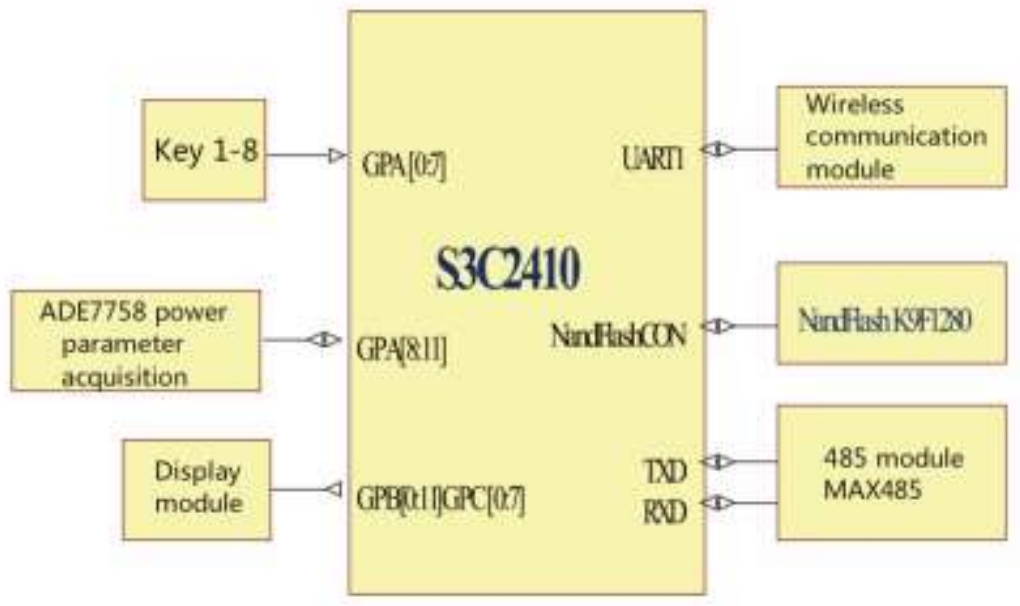

Figure.3 Basic peripheral circuit of microprocessors 
(3) Storage and expansion

The data measured by the smart meter are stored in the external expansion memory NandFlash chip K9F1208, and the 8-bit NANDFlash controller is integrated in S3C2410. K9F1208 is a large capacity and high reliable Flash memory that is produced by Samsung Corp in NAND technology. The device has a storage capacity of 64MB; in addition, there are 2048KB spare storage areas. The 8 bit I/O port uses address, data and command multiplexing method, which makes the interface circuit simple.

(4) Communication Interface

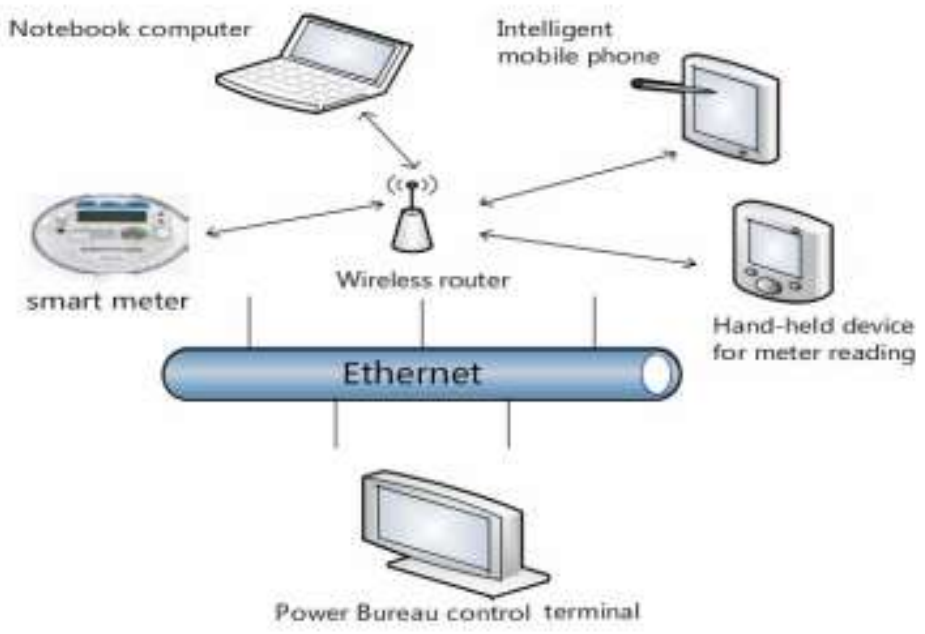

Figure.4 Schematic diagram of wireless network module

The design uses RS485 communication and WIFI wireless network module to communicate two communication interfaces. S3C2410 has three UART interfaces, and selects a USRT interface to handle data received and transmitted by RS-485 communication. The smart meter uses embedded UART-WIFI module to realize the function of transmitting power data in wireless network ${ }^{[4]}$. This function not only cancels line investment and reduces costs, especially in outdoor or outdated buildings where it is difficult to install cable equipment. The wireless network module solves the difficult problem of installation. It is possible to set or read the meter data of the corresponding location directly by the power meter covered by the local network on the host side. Another obvious advantage is the wide application of WIFI network in consumer electronics. In the future, smart terminals such as mobile phones or tablet computers can be used to access the meter data and check the electricity information at any time. This will bring great convenience to life and truly smart home. The schematic diagram of the wireless communication structure is shown in Figure 4. 


\section{Software Design}

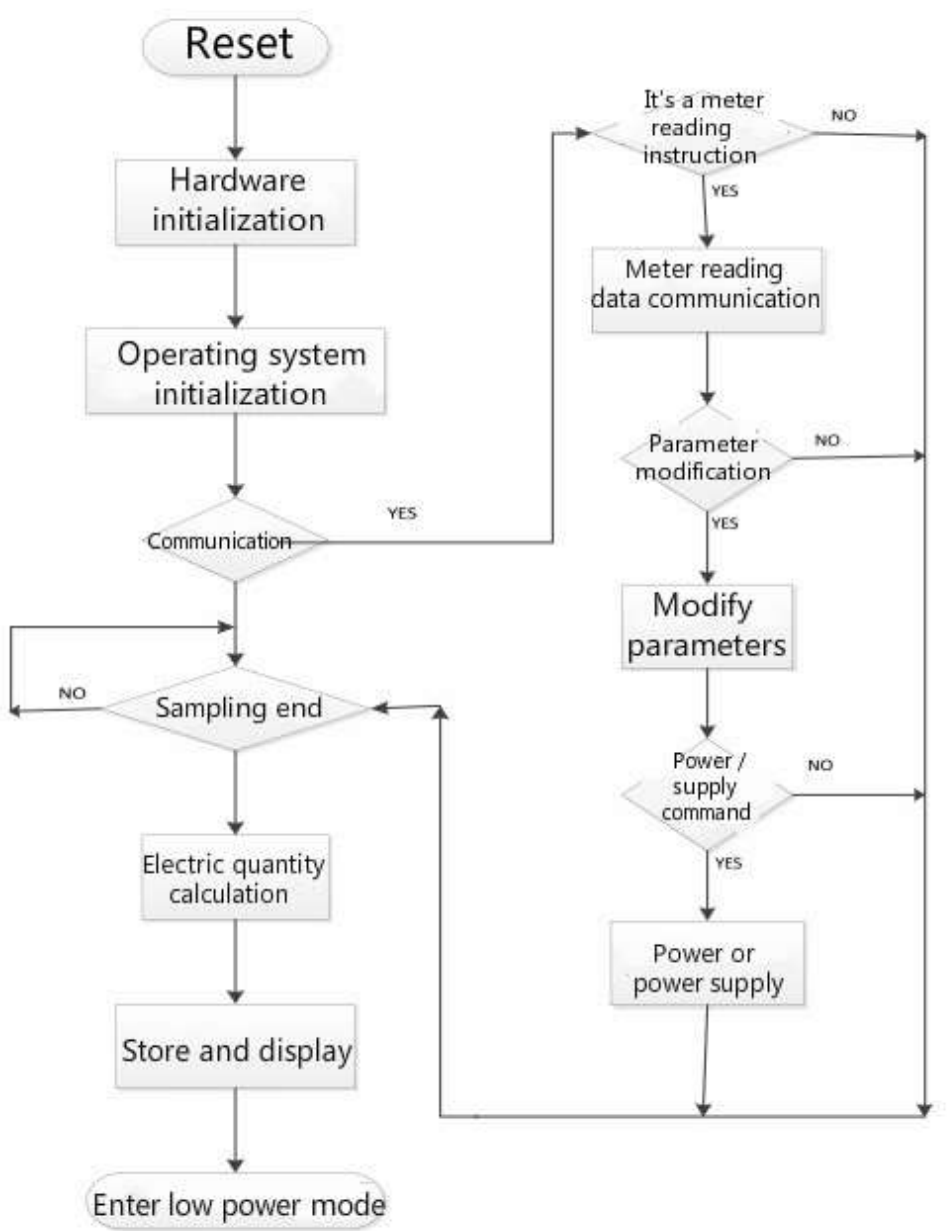

Figure 5. System software flow chart

The operation process of the meter system is as follows: first, hardware initialization and embedded Linux operation system initialization, then enter user task processing. On the one hand, the interrupts from the electric energy metering chip are handled at any time, and the quantity of electricity is calculated and stored after the detection. On the other hand, it can detect whether the upper computer sends the meter reading instructions at any time. After the detection, the corresponding processing is carried out and the communication is carried out through the WIFI communication module. The system software flow chart is shown in Figure 5.

\section{Summary and Prospect}

The electric energy meter currently on the market is mainly an electronic energy meter, which solves the problem of the lack of precision in energy metering of mechanical watches in the past, and along with the continuous development of the Internet of things technology, smart terminals (smartphones, tablet computers, etc.) are widely present. If the meter can be connected to a WIFI network, it is easier to read the consumption data and to allocate a reasonable amount of electricity. This article focuses on the hardware construction of the smart meter and the software driver implementation method. There are many issues that do not involve the details, such as energy-saving technology, data transmission methods and network address settings. The author will conduct further research.

\section{Reference}

[1] Manbachi M, Farhangi H, Palizban A, et al. Smart grid adaptive energy conservation and optimization engine utilizing Particle Swarm Optimization and Fuzzification[J]. Applied Energy, 2016, 174:69-79. 
[2] Hurtado L A, Nguyen P H, Kling W L. Smart grid and smart building inter-operation using agent-based particle swarm optimization[J]. Sustainable Energy Grids \& Networks, 2015, 2:32-40.

[3] Male S, Vethekar P. A Smart Wireless Electronic Energy Meter Reading Using, Embedded Technology[J]. International Journal of Engineering Research \& Applications, 2014, 4(1).

[4] Wang Z, Zhang K. Energy meter monitoring sensor network technology research[C]// China International Conference on Electricity Distribution. IEEE, 2011:1-7.

[5] Yu D, Peng L. When does Inferring Reputation Probability Countervail Temptation in Cooperative Behaviors for the Prisoners' Dilemma Game? [J]. Chaos, Solitons \& Fractals, 2015, 78: 238-244.

Ning Li; February 1968; male; Han nationality;Urumqi,Xinjiang Uygur Autonomous Region; Bachelor degree;Senior engineer;Research direction:Electric energy measurement technology 